\title{
Head Gesture Recognition System Using Gesture Cam
}

\author{
Rushikesh T. Bankar ${ }^{1}$ and Suresh S. Salankar ${ }^{2}$ \\ Research Scholar, \\ ${ }^{1}$ Department of Electronics Engineering, \\ G. H. Raisoni College of Engineering, \\ Nagpur, India. \\ ${ }^{2}$ Department of Electronics \& Telecommunication Engineering, \\ G. H. Raisoni College of Engineering, \\ Nagpur, India. \\ rushikesh.bankar@raisoni.net ${ }^{1}$, suresh.salankar@raisoni.net ${ }^{2}$
}

\begin{abstract}
Head Gesture means the position made by the movement of head by considering all its facial geometry as eyes, nose, lips etc. The use of such gesture is to express thought, emotion, etc. Such Head Gesture is very useful / beneficial for the Handicapped / Peoples having Paralysis from neck onwards. For such peoples Hand Gesture Recognition System is not useful. Such peoples give the indications by using their Head Movements. The gesture recognition from the video sequences is one of the most important challenges in the computer vision. It offers to the system, the ability to identify, recognize and interpret the human gestures in order to control some devices. In this paper, we provide a review on gesture recognition with particular emphasis on Head Gestures and Facial Expressions. Applications involving Face Detection, Face Tracking, Gesture Recognition, and Obstacle Detection are discussed in detail.
\end{abstract}

Keywords: Face Recognition, Intelligent Systems, Computer Vision, Head Gestures, Face Detection

\section{Introduction}

The evolution of the user interface witnessed the development from text based on keyboard to graphical user interface based on mice. In current virtual environments applications, joysticks, mice, and keyboards are the popular, dominant as well as useful devices. The use of head gestures has become an important part of Human Computer Intelligent Interaction in the recent years, which plays an important role for research in modelling, analysing and recognition of head gestures. Many techniques developed in human computer intelligent interaction can be extended to other area like robot control.

A Smart Camera in this proposed system can be defined as a vision system to produce a high level processing of the images scene and accordingly that it should generate application specific data or information in which the user requires, to be used in an autonomous as well as an intelligent system. The smart camera consists of a processing unit. To perform the application specific information processing, the processing unit which is, in the smart camera. The application specific information processing unit is used to extract the information from the captured images of the user for an application purpose. For example, a motion triggered camera captures the video of the user's head, detects the movements of the user's head and gives an indication for the face direction.

To recognize the gestures like head gesture, hand gesture or arm gesture, is a complex task which involves many aspects such as motion modelling, motion analysis, pattern recognition and machine learning. Since gesture recognition is receiving more attention in 
existing research, a comprehensive review on various gesture recognition techniques such as hand gesture, head gesture or arm gesture developed in recent years is needed.

A smart camera which is based on PC, the camera is a webcam, of which the output of the webcam that is the video output is connected to a PC port through Fire wire, or the other protocols. Such kind of camera configuration has a few limitations. For example, a general purpose $\mathrm{PC}$ is not suitable to the intensive image processing of the high resolution. Also the requirement of the bandwidth for the PC camera link is to be very high. The Gesture Cam that is the Smart Camera can greatly simplify the application system design to perform image processing tasks. The Gesture Cam / Smart Camera having the embedded processing unit, is a much better way to process the images at the high resolution as well as high frame rate. This performs in the real time. The only image feature or the high level description of the user's face in four directions is to be transferred to the computer, is the output of the smart camera, the bandwidth is low.

The smart camera or gesture cam, can have many applications, such as computer vision, security, video surveillance, human computer interface, user interface technology is an integral as well as critical part of the most modern ICT systems. Unlike all existing User Interface Technologies which are computer centered, an MMUI that is Multi Modal User Interfaces is user centered. The Multi Modal User Interfaces allows a user or the disabled people to interact with a computer using user's natural communication modalities, such as speech, Gestures, Eye Gaze, and Facial Expression. The multi modal user interface that is MMUI systems include new devices as well as improvements in the Recognition Based Technologies. For example, Speech Recognition, and Gesture Recognition. Such systems are more robust as well as stable manner than the unimodal recognition system which involves the single recognition based technology.

The successful deployment of the intelligent system relies on their high performance as well as low cost. As compared to the other head gesture recognition system, the main performance of the intelligent system includes the autonomous navigation capability for good safety, flexibility, mobility, obstacle avoidance etc., an intelligent system including voice based control system, vision based control system, and sensor based control system. The new generation, an intelligent system of head gesture recognition should be able to deal with the uncertainties from the practical applications point of view. They are the user head either out of the image view or only the profile face is in the captured image, the face colour of the user is user dependent, or may change dramatically in the varying illumination conditions, the different facial appearances conditions of the user, such as mustache and glasses, and the cluttered background. The proposed system is an intelligent system for the head gesture recognition, which is based on the combination of adaboost and improved camshift algorithms.

The research on human gestures for the disabled peoples is going on. K. Yuan [1] represents the system for an intelligent wheelchair in which the head gesture is recognized. This system is a hands free control system. In this paper, the real time face detection, face tracking and gesture recognition is proposed, namely Head Gesture Based Interface (HGI). It is to be used as the Human Robot Interface for the Intelligent Wheelchair, namely Robo Chair. The system is used to solve the problems. They are - The user head may be out of the image view, or only the profile face is in the captured image, the face color is user dependent, and may change dramatically in varying illumination conditions, the user may have different facial appearances, such as mustache and glasses, and the background may be cluttered when an Intelligent Wheelchair move in the real world. H. Kim, S. H. Lee, M. K. Sohn and D. J. Kim [2] present a system to estimate the head pose in the gray level means black \& white images. The two techniques were employed in this research project. The method of Random Forests was employed for dealing with the large set of training data. This algorithm is used in the computer vision System. To detect the user's faces in the varying illumination conditions, a Binary Pattern Run Length matrix is used. This matrix also includes a Binary Pattern. M. Davy and R. Deepa [3] present Head Movement 
System using Accelerometer Sensor. The gesture generated by the movement of the head, which can be performed by the handicapped peoples or users or disabled peoples whose body parts below the neck is paralyzed. The system includes the accelerometer sensor which detects the head movement of the user or disabled people. The proposed system uses the accelerometer to find out the movement of the head is detected. Ericka Janet Rechy Ramirez and Huosheng $\mathrm{Hu}$ [4] present a flexible bio signal based HMI system. In this HMI system, an Emotive EPOC sensor is used for the head movements of the user. The sensor recognizes the head movements of the user. Chanlit Noiruxsar and Pranchalee Samanpiboon [5] presents face orientation recognition system for head gesture recognition system. The USB camera was fixed in front of the user's face. The face of the user was detected using adaboost algorithm. The face of the user in the four different directions, which is used for generating the control commands for the wheelchair or motion control system, consists of front direction, right side direction, left side direction, upside direction and down side direction. The system in which, the movement of robo chair can be controlled by using head gesture is presented in [6]. In this system, a web cam is used for the input of the user. A cascade object detection method is used for the face detection of the user. Rushikesh T. Bankar and Suresh S. Salankar [10] present an Intelligent Head Gesture Recognition System. The system is useful for the handicapped peoples.

The gesture (Hand, Head, Arm, Eye etc.) recognition is an important part of Multi Modal User Interfaces system. The vision based gesture recognition system involves the general purpose camera. This general purpose camera is connected to the general purpose PC for processing the proposed system.

\section{Design of Gesture Cam}

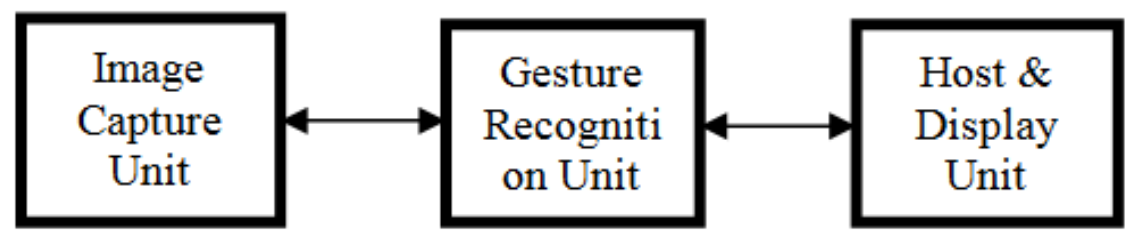

Figure 1. Gesture Cam Design Components.

Figure 1 shows the design of a Gesture Cam. The Gesture Cam is the FPGA based Smart Camera which can perform all the processing tasks. The use of FPGA as a development platform is because as compared to PC which performs the video processing tasks in real time, the FPGA is a better computing platform. The high bandwidth requirement between the camera and PC is not necessary because the camera output can be as simple as an index of a gesture among the predefined head gesture data base.

The gesture cam consists of the three parts. The first part is the image capture unit. The image capture unit consists of a small in-house built PCB. A megapixel CMOS color image sensor OV9620 is to be mounted on the PCB from the omnivision. The PCB is to be fitted into a dummy camera casing.

The second part is the Gesture Recognition Unit. To form Gesture Recognition Unit, an FPGA development kit has been chosen. The kit is a powerful development platform for the imaging applications.

The third part is the host $\&$ display unit. Inside the host $\&$ display unit, there consists of a host PC. Also there are the two LCD monitors. The first LCD Monitor is used for the code development as well as user interface for the camera configuration. The second LCD Monitor is used for the real time video display of results from the different stages of processing. 
The output of the Gesture Cam is interfaced with the Microcontroller for the movement of the wheelchair or the generation of the motion control commands.

\section{Face Detection Method}

For detection of user's face, we use the viola / jones face detector method. This is a widely used method for the real time object detection. In this method, the training is slow but the detection is very fast.

Before applying the viola / jones face detection method, first we take the video of the user using web cam. From the input video, the user's images are grabbed. These grabbed images then processes using the viola / jones face detection method.

The face detection is done in the four directions. These directions are the left turn, right turn, forward, and reverse. When the user wants to turn the left, the face detection is done for left turn image of the user.

\section{Experimental Result}

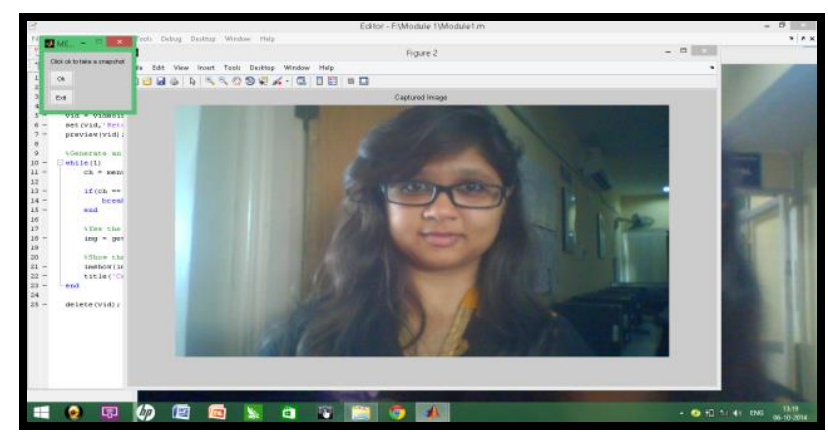

Figure 2. Grabbed Image of the User

Figure 2 shows the grabbed image of the user. First we take the input video of the user. From this input video, the user's input images are grabbed. These grabbed input images are used for the further processes.

In further process, the viola / jones face detector method is used. The above grabbed images are in four directions of the user. The four directions are turn left of the user's head, right turn, forward, and reverse direction.

The viola / jones face detector method is applied for these four directional images of the user.

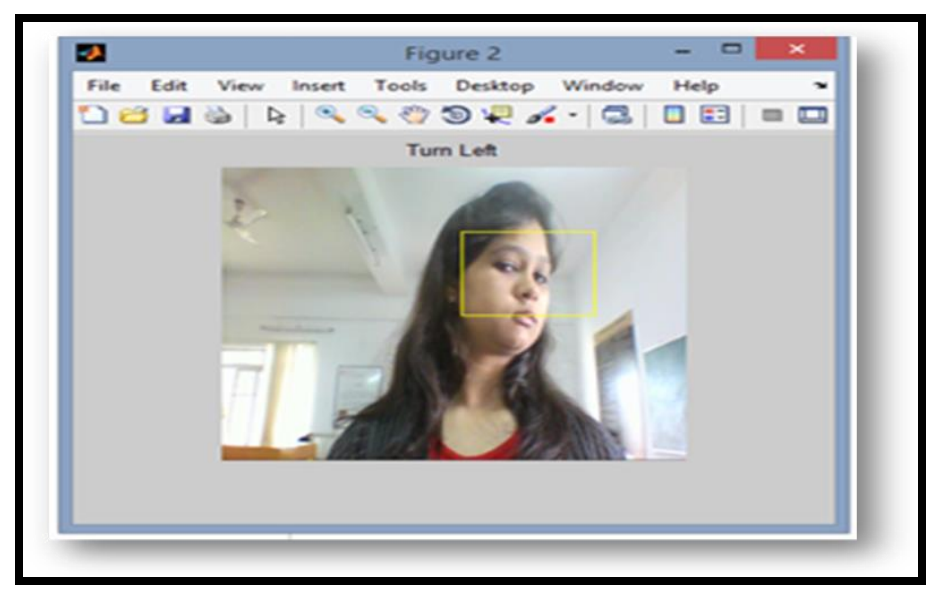

Figure 3. Face Detection of the User 
Figure 3 shows the face detection of the user. The face detection of the user is done in the four directions, left, right, forward, and reverse. The left turn profile of the user is detected which is shown in the figure 3. The viola / jones face detection method is applied for the left turn of the user's head.

\section{Obstacle Detection Technique}

The proposed system is mainly adapted for the people who are suffering with different disabilities. For those peoples, in certain conditions, problems could be occurred while handling the devices, during certain conditions. The system includes the obstacle detection technique. The system is responsible for the detection of obstacles while performing the proposed system. The subsystem consists of an ultrasonic sensor. The sensor emits the sound waves. The frequency of the sound waves is well above the perceivable frequency range of the human ears. The sound waves which emits from the sensor, when strike an object, it gets reflected. They are received by the sensor. In our system, a limit of $40 \mathrm{~cm}$ was set. If any obstacle comes closer than this limit, a buzzer activates. In this way the user is warned.

\section{Conclusions}

We have presented the head gesture recognition system using gesture cam. The gesture cam is the FPGA based smart camera. This FPGA based smart camera can recognize the head gestures. The output of the gesture cam is then interfaced with the microcontroller for further taking action to the wheelchair for turning as per the need of the user or generating the motion control commands. The intelligent system for head gesture recognition using gesture cam is completed when it is implemented on the wheelchair.

The next work is to design the Gesture Camera for the further processing. The face detection in four different directions is to be done. For this face detection, the web cam is used. After designing the gesture cam, the system is an intelligent system to recognize the human head gesture for the disabled peoples.

\section{References}

[1] P. Jia, H. Hu, T. Lu and K. Yuan, "The Hands Free Control of an Intelligent Wheelchair Using Head Gestures," an International Journal 34 / 1 (2007) 60 - 68.

[2] H. Kim, S. H. Lee, M. K. Sohn and D. J. Kim, "The head pose estimation in varrying illumination conditions", Human Centric Computing and Information Sciences, a Springer Open Journal, 2014.

[3] Manju Davy and R. Deepa, "The Head Movement Using Accelerometer Sensor,” International Journal of Applied Sciences and Engineering Research, Volume - 3, Issue - 1, 2014.

[4] Ericka Janet Rechy Ramirez and Huosheng Hu, "An Electric Powered Wheelchair For Hands Free Control Using a Flexible Bio Signal Based HMI," An International Journal of Artificial Life Research, 4 (1), 59 - 76, January - March 2014.

[5] C. Noiruxsar and P. Samanpiboon, "An Electric Wheelchair Control Using Face Orientation,” Journal of Automation and Control Engineering, Volume - 2, No. 4, December - 2014.

[6] Shraddha V. Manikpure, Rushikesh T. Bankar and Suresh S. Salankar, "The control of robo chair using head gesture recognition", International Journal of Innovative Science and Modern Engineering (IJISME), ISSN: 2319 - 6386, Volume - 3, Issue - 2, January - 2015.

[7] Pei Fia and Huosheng H. Hu, "The movement of an intelligent wheelchair based on head gesture recognition," Research Article, www.emeraldinsight.com/0143 - 991X.htm..

[8] Parimita Saika and Karen Das, "Head Gesture Recognition Using Optical Flow Based Classification with Reinforcement of GMM Based Background Subtraction", International Journal of Computer Applications (0975 - 8887), Volume - 65, No. 25, March - 2013.

[9] Preeti Srivastava, Dr. S. Chatterjee and Ritula Thakur, "A Novel Head Gesture Recognition Based Control For Intelligent Wheelchairs", International Journal of Research in Electrical \& Electronics Engineering, Volume - 2, Issue - 2, April - June, 2014, PP. 10 - 17.

[10] Rushikesh T. Bankar and Suresh S. Salankar, "Implementation of an intelligent wheelchair using head gesture", International Journal of Innovative Science and Modern Engineering (IJISME), ISSN: 2319 6386, Volume - 3, Issue - 2, January - 2015. 
[11] Yuan Luo Zhang Fang Hu and Lin Li Yizhang, "A Novel Head Gesture Recognition Means in the Human Wheelchair Interaction", International Conference on Computer Application and System Modelling (ICCASM - 2010).

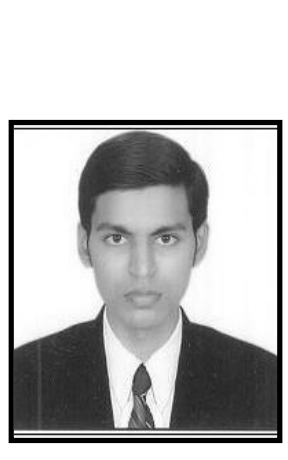

\begin{abstract}
Authors
Rushikesh T. Bankar, he is a Research Scholar, Ph. D Pursuing Student, in the Electronics Engineering Department at the Research Center, G. H. Raisoni College of Engineering, Nagpur (An Autonomous Institute under the UGC Act 1956), India. E-Mail: rushikesh.bankar@raisoni.net. His Professional Interests focus on Image Processing, Soft Computing, and Intelligent Transportation System. He is a member of IEEE.
\end{abstract}

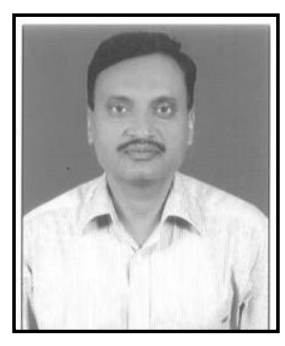

Suresh S. Salankar, Professor, G. H. Raisoni College of Engineering, Nagpur, India. E-Mail: suresh.salankar@ raisoni.net. Ph. $D$ in Electronics Telecommunication Engineering from SRTM University, Nanded, India in 2008. His research interests are in the design and evaluation of Learning Algorithms for Pattern Recognition Applications. This includes, in particular, Neural Network Classifiers, Support Vector Machines Classifier and Classifier Combing Strategies. He has published several papers in these areas. He is a member of the Institution of Engineers, India, Member of the Institution of Electronics \& Telecommunication Engineers (IETE), and Member of IEEE \& Indian Society for Technical Education (ISTE). 